Original Article

\title{
LATE-STAGE PRESENTATION OF TESTICULAR CANCER PATIENTS ATTENDED A SPECIALIZED HOSPITAL: FINDINGS OF A CROSS- SECTIONAL STUDY
}

\author{
Karim AM.M.N $N^{I}$ Arman K. $A^{2}$, Khurshid $N^{3}$, Afroz $T^{4 *}$
}

\begin{abstract}
Background: Testicular Cancer (TC) is of interest and importance because its incidence has been increasing in most countries over the past four decades. The purpose of this study is to determine the frequency of patients with common histopathology, most common stage during presentation, biochemical and radiological findings, common levels of tumour markers.
\end{abstract}

Methods: This cross-sectional study was carried out in the Department of Medical Oncology of National Institute of Cancer Research and Hospital (NICRH), Mohakhali, Dhaka, Bangladesh from July 2015 to June 2016. A total number of 52 patients were taken as study participants who are admitted as newly diagnosed at the NICRH. Data collection: tumour markers, USG of whole abdomen and chest X-ray were performed to all the patients for determination of the staging of the tumour.

Results: The present study found the majority of the patients were found in stage III disease. Patients with Cryptorchidism needed to be educated regarding its early management to reduce the incidence of testicular tumour among them. It was observed that young people could be adequately knowledgeable and optimal awareness could be developed regarding early symptoms of the disease, then they may be found in earlier stage of their disease, which is curable with modern management method. Targeting to alter the cancer patients' presentation by rising consciousness about TC, prognosis can be largely improved in future.

Conclusion: This study was conducted to find out the features which were unknown in perspective of Bangladesh, so that burden of TC can be reduced, and prognosis can be further improved of this potentially curable disease.

JOPSOM 2021; 40(1): 26-33

https://doi.org/10.3329/jopsom.v40i1.56688

Keyword: Testicular Cancer, Pathological findings, Staging, Young adult, Bangladesh.

1. Dr. Abul Masud Md. Nurul Karim, MBBS, MPH, Assistant Professor, Department of Epidemiology, NIPSOM, Mohakhali, Dhaka-1212; Email: dnurulkarim@yahoo.com

2. Dr. Kazi Abdullah Arman, MBBS, MD, Registrar, Medical Oncology, Evercare Hospital, Dhaka- 1229, drkaziarman@gmail.com

3. Dr. Narita Khurshid, MBBS, MD, Registrar, Evercare Hospital Dhaka-1229; naritabd24@yahoo.com

4. Dr. Tamanna Afroz, MBBS, MPhil, dr.tamannaafroz@gmail.com

*Correspondence: Tamanna Afroz, Mob: +8801812165022,dr.tamannaafroz@gmail.com

\section{INTRODUCTION}

Testicular Cancer (TC) is the commonest solid neoplasia, which is approximately $1 \%-1.5 \%$, in men aged 20-40 years [1]. It is the solid organ malignancy among young male with overall 10 years relative survival rate in more than $95 \%$ cases [2]. The prognosis of the disease highly depends on the stage of presentation. In spite of the importance, in Bangladeshi context, there is scarcity of research on testicular tumour, especially histopathology, biochemical and radiological findings, levels of tumour markers and most common stage of presentation. If all these features are known to us, the outcome of the disease can be positively influenced by educating people and developing awareness among them. Therefore, this research was conducted to study the commonest stage during presentation, and pathological characteristics of testicular tumour in adolescent and adult. 
On average, testicular cancer (TC) represents the most common nonhematologic malignancy in men between 15 and 49 years old. Younger males are $1-1.5 \%$ affected by this solid tumour, which has good prognosis and is totally curable if diagnosed in an early stage [4]. Nonetheless, the testicular malignancy has one of the most complete and thorough staging systems, which combines clinical, pathologic, radiologic, and serum tumour marker components. Proper staging facilities gives the opportunity to make a management plan followed by adequate surveillance for these patients. International classification on histological features based on laboratory support is important to document for treatment success. With the medical advancement in Bangladesh, it is adequately possible to successfully manage testicular carcinoma among young male. Although, there is not enough empirical studies done on TC till dates to accumulate in development of clinical and preventive measures for affected group of people. This study on TC, at a tertiary medical centre for cancer in Dhaka, was done to describe the staging of the cancer in regards of treatment availability with the laboratory evidence.

Testicular Cancer (TC) is of interest and importance because its incidence has been increasing in most countries over the past four decades. The association between some risk factors, including cryptorchidism, a previous history of testicular cancer and a family history of testicular cancer, and the incidence of testicular cancer has been widely reported, with TC [3].

Classification: Testicular tumours can be categorized into a) Germ Cell Tumour (GCT) and b) Non-Germ Cell Tumours (NGCT). Approximately 95 percent of malignant tumours arising in the testis are GCT, a term that indicates their origin in primordial germ cells. GCT also occasionally arise in extragonadal primary sites, and their management follows that of testicular germ-cell tumours. More than 90 percent of patients with newly diagnosed GCT are cured, and delay in diagnosis correlates with a higher stage at presentation for treatment $[5,6]$. Non-germ cell primary tumours of the testis derive from the sex cords (Sertoli cells) and stroma (Leydig cells). These tumours are malignant in only $10 \%$ of cases. Nonprimary tumours such as lymphoma, leukaemia, and metastases can also manifest as testicular masses. In the 2016, the updated version of the WHO Classification of Germ Cell Tumours (GCT) explained the characteristic of Testicular Cancer (TC) with histological explanation [7]. Germ-cell tumours are classified as seminomatous or non-seminomatous, reflecting their origin in primordial germ cells and their remarkable ability to differentiate in vivo.
Histopathological findings: Most non-seminomatous germ-cell tumours include multiple cell types, and seminoma may be a component. When seminomatous and non-seminomatous areas are both present in a tumour, management should follow that for nonseminomatous tumours, since these tumours are more clinically aggressive. Therefore, a tumour is diagnosed as a seminoma only if the histologic results show pure seminoma and if the serum concentration of alphafetoprotein, a marker of non-seminomatous tumours, is normal. The NSGCT as Embryonal carcinoma, Yolk sac tumour, Teratocarcinoma, Choriocarcinoma has mixed histopathology and whether they are combination of two or more NSGCTs or combination of Seminoma and NSGCTs, could be also possible. The incidence of NSGCT peaks in the third decade of life. These tumours are composed of embryonal carcinoma, teratoma, choriocarcinoma, and yolk-sac carcinoma (endodermal-sinus tumour) cell types. Embryonal carcinoma is the most undifferentiated cell type, with totipotential capacity to differentiate to the other non-seminomatous cell types [7].

a) Seminoma: Seminoma is the most common pure germ cell tumour. It accounts for $35 \%-50 \%$ of all germ cell tumours and are most frequent in the fourth decade of life. There may be a high mitotic rate (anaplastic), syncytiotrophoblastic giant cells, and an increased serum concentration of human chorionic gonadotropin (hCG), but these do not influence management [8]. Spermatolytic seminomas rarely metastasize, and they present almost exclusively in elderly men [9]. At histologic analysis, the cellular morphology of seminomas resembles that of primitive germ cells. The cells are relatively uniform with clear cytoplasm and an associated lymphoid infiltrate. Bilateral tumours are also rare, occurring in $2 \%$ of patients, and are almost always asynchronous [3].

\section{b) Non-seminomatous Germ Cell Tumours (NSGCT)}

Embryonal Carcinoma: Embryonal carcinoma is the most undifferentiated cell type, with totipotential capacity to differentiate to the other nonseminomatous cell types. Embryonal carcinoma may produce elevated serum concentrations of hCG, alphafetoprotein, or both. Embryonal carcinoma is present in $87 \%$ of MGCs, although in its pure form, it accounts for only $2 \%-3 \%$ of all testicular tumours. Embryonal carcinoma occurs in a younger population than does seminoma, as it is seen most often in men aged 25-35 years [4]. Treatment for all the non-seminomatous tumours is similar [10].

Yolk-sac tumours: Yolk-sac tumours (YST) mimic the embryonic yolk sac histologically and nearly always produce alpha-fetoprotein. Pure yolk-sac 
histologic findings are frequently present in germ-cell tumours arising in the mediastinum. Yolk sac tumours account for $80 \%$ of childhood testicular tumours, with most cases occurring before the age of 2 years [11]. In its pure form, yolk sac tumour is present in $44 \%$ of adult cases of mixed germ cell tumour. AFP is normally produced by the embryonic yolk sac, and thus serum $\alpha$-fetoprotein levels are elevated in greater than $90 \%$ of patients with yolk sac tumour [11].

Teratoma: Generally, teratomas occur in children less than 4 years of age. In its pure form, teratoma is rare in adults; however, teratomatous elements occur in approximately half of all adult cases of mixed germ cell tumour ${ }^{[11,12]}$. Teratoma is composed of somatic cell types from two or more germ-cell layers (ectoderm, mesoderm, or endoderm). Mature teratomas consist of adult-type differential cell types, such as cartilage or mucin-producing glandular epithelium. Immature teratomas are tumours with partial somatic differentiation, similar to that seen in a foetus. On rare occasions, a teratoma develops aggressive growth and histologically resembles a somatic cancer such as rhabdomyosarcoma, adenocarcinoma, or primitive neuroectodermal tumour ${ }^{[12,13]}$. This is called a teratoma with malignant transformation, and it may occur in the setting of a germ-cell tumour arising from any primary site ${ }^{[13]}$. These tumours are subclassified into mature, immature, and those with malignant areas [11]. The biologic behaviour of teratomas is quite variable, depending on the pubertal status of the testis. In prepubertal testes, pure teratomas are considered benign even when they are histologically immature ${ }^{[3,}$ ${ }^{4,10]}$. Of important distinction, every element in a postpubertal testicular teratoma (mature or immature) can metastasize, irrespective of its histologic characteristic. Furthermore, the metastases may contain non-teratomatous germ cell elements. So, most author emphasized that mature teratoma should not be equated with benignity ${ }^{[10]}$.

Choriocarcinoma: Choriocarcinoma is a rare germ cell tumour. In its pure form, it is seen in less than $1 \%$ of patients, but it occurs in mixed germ cell tumours in $8 \%$ of cases and is usually associated with widely metastatic disease and high serum concentrations of BhCG. It develops in patients in the $2 \mathrm{nd}$ and $3 \mathrm{rd}$ decades of life and is a highly malignant tumour composed of an admixture of cytotrophoblast and syncytiotrophoblastic cells. Often, there is early widespread metastasis, and patients may present with symptoms referable to their metastases rather than a palpable testicular mass. The levels of human chorionic gonadotropin (hCG) are elevated and cause gynecomastia in $10 \%$ of cases. Choriocarcinoma has the worst prognosis of any of the germ cell tumours, with death usually occurring within 1 year of diagnosis [3]. Patients with mixed germ cell tumours with choriocarcinoma fair better than those with pure choriocarcinoma tumours, but a very high level of hCG $(>50,000 \mathrm{IU} / \mathrm{L})$ portends a poor prognosis with a 5 -year survival rate of $48 \%$ [12].

Mixed germ cell tumours: Mixed germ cell tumours (MGCT) contain more than one germ cell component. Of the non-seminomatous germ cell tumours, MGCT are much more common than any of the pure histologic forms and represent 32\%-60\% of all GCT. Embryonal carcinoma is the most common component and is often combined with one or more components of teratoma, seminoma, and yolk sac tumour. Notable fact is, the average age of presentation for patients with MGCT is 30 years ${ }^{[10]}$. Here, the imaging findings are variable, reflecting the diversity of this group of tumours.

So far, seminoma is extremely radiosensitive, and its treatment is a true success story. Radiation therapy for low-stage tumours is very effective, resulting in a 5year survival rate of $95 \%$. Patients with advanced stage disease receive chemotherapy, which may be followed by radiation therapy for curative management ${ }^{[10]}$. On the contrary, non-seminomatous tumours are not as radiosensitive as seminomas; therefore, the patient may receive chemotherapy rather than radiation therapy as part of the treatment protocol. After treatment, serial CT scans often show a decrease in the attenuation as well as the size of retroperitoneal masses [14]. A MGCT or immature teratoma may evolve into a mature teratoma too ${ }^{[13]}$. Resection of these treated masses shows that approximately $40 \%$ have undergone necrosis or fibrosis, whereas $40 \%$ have evolved into mature teratoma ${ }^{[12]}$. The remaining $20 \%$ will have areas of residual tumour. A mature teratoma can grow despite maintaining a benign histologic type (growing teratoma syndrome), or, as previously emphasized, it can transform into a more aggressive histologic type ${ }^{[12]}$.

\section{Staging based on radiology and biochemistry findings:}

Staging of the TC depends on surgical pathology of the orchiectomy specimen, tumour markers before and after orchiectomy (AFP, HCG, and LDH), chest radiography or chest $\mathrm{CT}$, and $\mathrm{CT}$ of the abdomen and pelvis. Depending on the clinical symptoms and presence of adenopathy on abdominal-pelvic CT, a staging $\mathrm{CT}$ is done or other bone and brain evolution is warrant. The staging systems commonly used are a) American Joint Committee on Cancer (AJCC) and b) International Germ Cell Tumour Consensus Conference Classification. The AJCC staging is a TNMS-based $(\mathrm{T}=$ tumour, $\mathrm{N}=$ node, $\mathrm{M}=$ metastases, 
$\mathrm{S}=$ serum markers) classification, because the progression of the tumour can also be monitored by the level of specific serum markers: AFP, HCG and LDH. Whereas surgical pathology determines the $T$ category of the neoplasm, imaging plays a critical role in determining the $\mathrm{N}$ and $\mathrm{M}$ components of testicular tumour staging. $\mathrm{N}$ category is determined by the extent of retroperitoneal lymphadenopathy, and M category describes distant metastases. Roman numeral stage grouping divides testicular cancer into three major groups on the basis of TNMS characteristics: tumours limited to the testis are stage I, those with retroperitoneal nodal involvement are stage II, and those with distant disease are stage III ${ }^{[15]}$.

\section{METHODS:}

Study design, period, settings, and population: The study was a cross-sectional type of observational study. The study was conducted during the period from July 2015 to June 2016. The study setting included Department of Medical Oncology of National Institute of Cancer Research and Hospital (NICRH), Dhaka, Bangladesh. Purposive sampling was done considering that the testicular tumour is comparatively rare among admitted patients. All the clinically suspected and diagnosed case of this tumour, at the age of 16 and above, admitted in the Department of Medical Oncology of NICRH, were selected as study population. The sample was collected by purposive sampling technique.

Recruitment procedure and data collection: All the patients were recruited as per inclusion and exclusion criteria. The relevant socio-demographic characteristics, clinical findings as well as the physical examination were performed after taking written consent from all the study subjects. Many of the patients came postoperatively and with a histopathology report. The histopathology reports were accepted, besides CXR, USG of whole abdomen or CT scan of abdomen was done along with the tumour markers e.g., AFP, $\beta \mathrm{hCG}$ and $\mathrm{LDH}$, prior to start treatment in this hospital.

Selection criteria: Newly diagnosed case of testicular tumour by histopathology, sonology or biochemistry were selected for the study. Male patient aged from 16 years or more having testicular tumour, suspected patients of testicular tumour who were confirmed later on as testicular tumour, and patients willing to participate were incuded in the study. Patients with extra gonadal germ cell tumours, already treated, having another primary tumour elsewhere in the body, and unable to provide consent were excluded.

\section{Data collection:}

All the patients were recruited as per inclusion and exclusion criteria, who came to NICRH for treatment. The relevant socio-demographic characteristics, clinical findings as well as the physical examination was performed. Patient's histopathology report (if available) was recorded. If there was no orchidectomy or histopathological evidence, then the patient was sent for orchidectomy and/or histopathology depending upon the indication. For determining the staging following investigations records were documented: Histopathological variations were recorded; CT scan of abdomen and pelvis or USG of W/A; Chest X-Ray P/A and Lateral view or CT scan of chest; and Tumour marker: Serum AFP, Serum HCG, Serum LDH. Data were collected by using a structured data collection sheet. All other required data were collected from hospital registration; case reports, staging was done in accordance and recorded as well for determination of treatment purpose. In the last part of data collection, hospital registered data was rechecking for confirmation of staging of the tumour.

\section{Data processing and analysis:}

Statistical analysis of the study was done by IBM SPSS statistics for Windows version 22.0 (Armonk, NY: BM Corp) ${ }^{[16]}$ The results were presented in tables, figures, charts and diagrams. Confidence interval was considered at $95 \%$ level. Quantitative data were expressed as mean (M) and standard deviation (SD) and qualitative data were expressed as frequency distribution and percentage determination by SPSS.

\section{Ethical consideration:}

Prior to the commencement of this study, the protocol was approved by the ethical committee of NICRH, Dhaka. The aim of the study along with its procedure, risks and benefits of this study was explained to the respondent in easily understandable local language and then written informed consent was obtained from each. It was assured that all information and records would be kept confidential, and the participation was fully voluntary according to the Declaration of Helsinki (WMA, 1964) ${ }^{[17]}$.

\section{RESULTS:}

Following findings were found in this study:

a) Maximum patients of this disease were distributed in 26 to 30 years age group which was $26.92 \%$, on both side of this age group the frequency tailed off. Mean age was 32 years with SD 10.2. Cryptorchidism was the only known risk factor that had significant association with testicular tumour. 


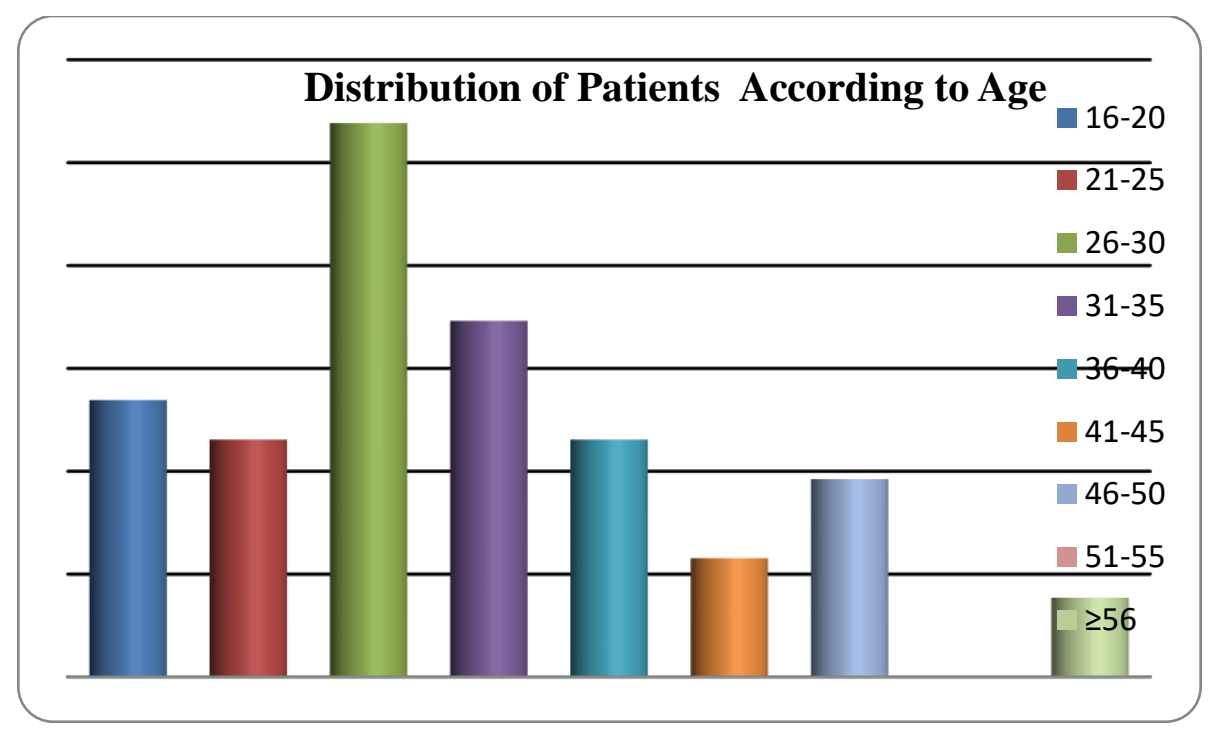

Figure 1: Bar diagram shows distribution of patients according to age.

b) The most common histopathology was Seminoma which was $38.46 \%$. Mixed germ cell tumour, Embryonal carcinoma, Teratocarcinoma and Yolk sac tumour was the histopathology of $23.08 \%, 21.15 \%$, $11.54 \%$ and $1.92 \%$ patients respectively. No patients came with Choriocarcinoma. Only 1 patient came with testicular sarcoma and 1 patient with metastatic mucinous adenocarcinoma which was $1.92 \%$ in both cases. Before starting the treatment $57.69 \%$ patients were in Grade 1 and $26.92 \%$ patients were in Grade 0 of ECOG performance score. By assessing most commonly found ECOG score we came to know that most of the patient could be able to receive the treatment and might continue it up to the end.

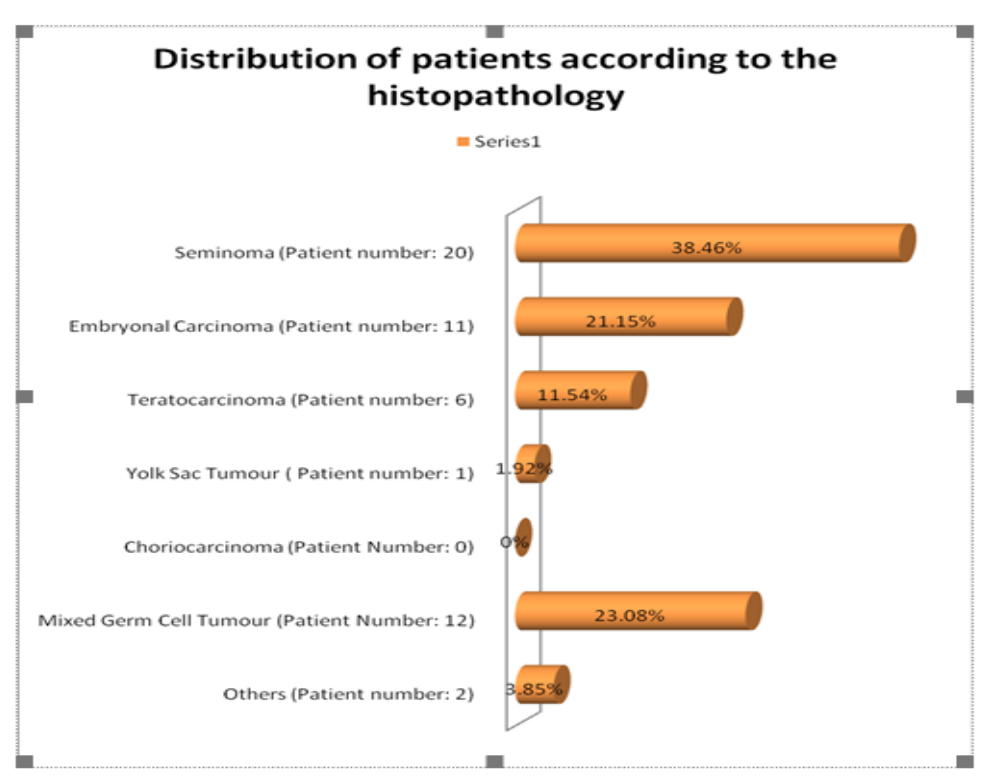

Figure 2: Bar diagram showing distribution of patients according to histopathology

c) Evaluating the symptoms and signs revealed most of the patients experienced swelling of the testis, diffuse testicular pain, back pain, costovertebral tenderness, painless testicular mass or lower abdominal pain and the duration from the appearance of the symptoms to diagnosis was pretty long.

d) Determining the stage at presentation revealed that $50 \%$ patients with germ cell tumour came with stage 
III disease, whereas $18 \%$ with stage I and $14 \%$ with stage II disease. Separately, in patients with Seminoma, stage I in $45 \%$, stage II in $20 \%$ and stage III was found in $35 \%$ of patients, in contrast in patients with NSGCT, stage I in 30\%, stage - II in $10 \%$ but stage III was found in as high as $60 \%$ patients. e) The significant part of this study is: in outer world, most of the patients come with stage I disease whereas in Bangladesh we found most of the patients came with stage III of testicular tumour.

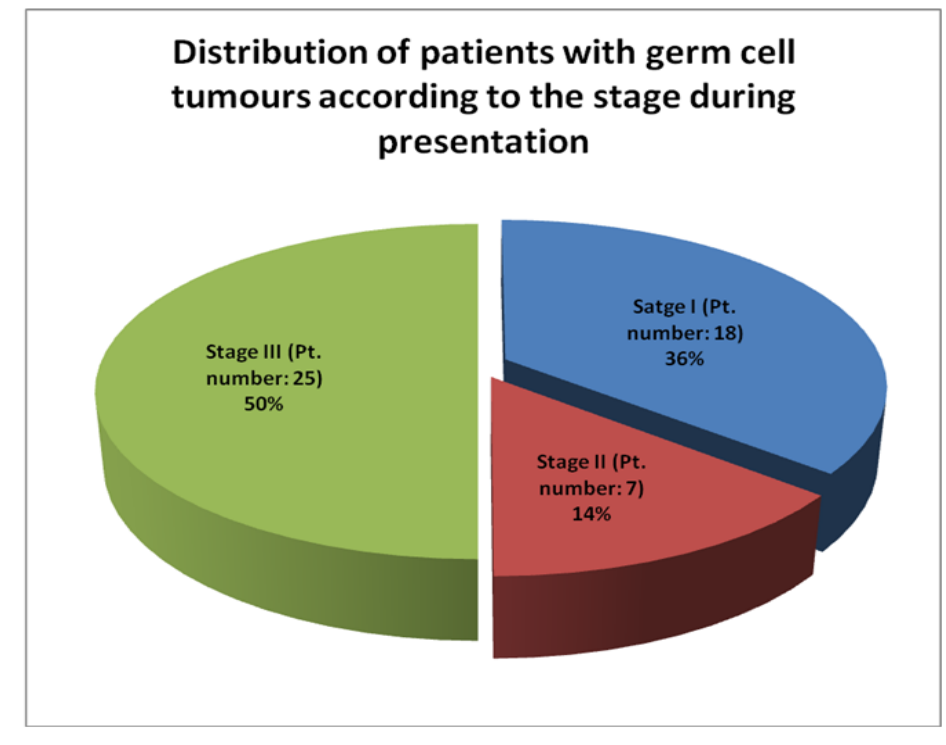

Figure 7: Pie chart showing distribution of patients with germ cell

tumours according to the stage during presentation

\section{DISCUSSION:}

The result of laboratory studies along with radiological findings, histopathology of the tumour, and stage of the disease and other relevant features of a disease is contributory in disease prevention and surveillance.

Radiology: Imaging studies play a vital role in the diagnosis and management of testicular cancer. Ultrasound is primarily used for initial diagnosis, and $\mathrm{CT}$ is the standard for cancer staging. MRI provides an equally powerful diagnostic alternative to CT for use in certain circumstances ${ }^{[14]}$.

Ultrasound: Ultrasound is not recommended for use in distant staging of testicular cancer because it is vastly inferior to $\mathrm{CT}$ or MRI in assessing the presence and burden of disease ${ }^{[14]}$.

CT: Although CT excels at identifying and determining the diameter of retroperitoneal lymph nodes, this modality cannot differentiate a lymph node that is infiltrated with malignant tissue from a lymph node that is benign. As a result, malignant lymph nodes are identified based on size criteria, with malignant nodes usually considered to be $8-10 \mathrm{~mm}$ or greater in diameter. Abdominopelvic CT offers sensitivity of approximately 70-80\%, but this is highly dependent on nodal size because testicular cancer has a high propensity for nodal micro metastases $7^{[14,18]}$.

MRI: Although abdominal CT offers excellent anatomic resolution of the retro peritoneum, the high dose of radiation generated and the relatively young age of testicular cancer patients have served as an impetus to evaluate MRI as a potential modality for retroperitoneal lymph node evaluation. Using CT as a reference standard, MRI has sensitivity of $97 \%$ and for experienced practitioners MRI serves as an excellent modality for retroperitoneal lymph node staging.

Chest Imaging: Because testicular cancer has propensity to spread to the mediastinal lymph nodes after reaching the retro peritoneum, chest imaging plays an important role in initial staging. CT of the chest represents the most sensitive evaluation, but this is compromised by reduced specificity with increased false-positive findings. Past research assessed the sensitivity and specificity of chest CT in initial staging of patients with seminoma and found that CT has a higher false-positive rate while maintaining the same sensitivity as radiography. Previous studies states, chest radiography is found preferable in patients with 
seminoma and patients with NSGCT with normal abdominal imaging ${ }^{[16]}$.

Brain Imaging: Imaging of the brain as part of initial staging is only recommended when there is a high suspicion for brain metastases, when the patient is neurologically symptomatic or has extensive pulmonary disease. Choriocarcinoma, the most aggressive histologic subtype, is most prone to metastasize to distant sites, and therefore brain imaging is indicated for patients with choriocarcinoma in their orchiectomy specimen and persistently elevated $\beta$-HCG. Three-dimensional imaging of the brain can be performed either with CT or MRI; with contrast administration to identify haemorrhage.

Tumour marker: In diagnosis of TC, reliable tumour marker is available, which made the disease management easier. For instance, Human chorionic gonadotropin (hCG), alpha fetoprotein (AFP), and lactate dehydrogenase $(\mathrm{ADH})$ play crucial roles in diagnosis, staging, prognosis, monitoring treatment response, and surveillance of seminomatous and nonseminomatous germ cell tumours. Herein we discuss the clinical applications of germ cell tumour markers ${ }^{[15]}$.

Over three quarters of patients with metastatic nonseminomatous germ cell tumours have a raised serum concentration of hCG or alpha-fetoprotein (AFP) or both. These markers may be used both as diagnostic aids and in monitoring the growth of the tumours. Elevated levels of AFP mean that a pure seminoma cannot be present. In contrast, about $20 \%$ of seminoma patients have mildly elevated levels of hCG/hCG-beta, which are synthesized in syncytiotrophoblastic giant cells and only rarely in inconspicuous rounded seminoma cells. Increased serum concentrations of $\beta$ hCG may be observed in both seminomas and nonseminomatous tumours. Increased $\beta \mathrm{hCG}$ concentrations are seen in 40 to 60 percent of patients with metastatic NSGCT, and 15 to 20 percent of patients with metastatic seminomas. Highly elevated $\beta$ hCG levels at the beginning indicate a poor prognosis regardless of other parameters and should be borne in mind when decisions on treatment are made. A third serum marker, lactate dehydrogenase, is less specific but has independent prognostic value in patients with advanced germ-cell tumours and it increases in approximately 60 percent of patients with NSGCT and 80 percent of those with SGCT. Nevertheless, LDH is of some value in the follow-up of marker-negative patients and can indicate a persistent tumour or a recurrence. Some authors have found evidence that initially elevated LDH may be an independent prognostic factor ${ }^{[18]}$. At least half the patients with, active seminoma have raised activities of serum placental alkaline phosphatase ${ }^{[19]}$. Based on the natural history of the disease, curability after multimodality treatment regimens is often declared after 5 years. However, relapse has been reported 10 years after treatment ${ }^{[20]}$. The existence of reliable testicular tumor marker has a significant impact on testicular cancer surveillance after primary therapy and aids in the early detection of disease recurrence. The frequency of tumor marker evaluation varies according to the tumor histology, stage, and previous therapy. Although crucial in the management of men with testicular cancer, current serum tumor markers do lack sensitivity and specificity.

\section{CONCLUSION:}

Treatment of TC is considered as an oncologic success story because, most individuals would be cured with a combination of surgery and chemotherapy. Laboratory diagnosis plays an important role in identifying tumours at patient presentation, accurately staging disease, and detecting recurrence during imaging surveillance and helps in treatment modalities.

\section{ACKNOWLEDGEMENT:}

The authors express the gratitude to Prof. Dr. Parveen Shahida Akhtar, Head of the Department, Department of Medical Oncology, National Institute of Cancer Research and Hospital (NICRH) Mohakhali, Dhaka, for the valuable instruction, constructive criticism, proper guidance, constant inspiration and all-round supervision during this research work.

\section{REFERENCE:}

1. Boccellino $\mathrm{M}$, Vanacore $\mathrm{D}$, Zappavigna $\mathrm{S}$, Cavaliere C, Rossetti S, D’Aniello C, Chieffi P, Amler E, Buonerba C, Di Lorenzo G, Di Franco R. Testicular cancer from diagnosis to epigenetic factors. Oncotarget. 2017 Nov 28;8(61):104654.

2. Milose JC, Filson CP, Weizer AZ, Hafez KS, Montgomery JS. Role of biochemical markers in testicular cancer: diagnosis, staging, and surveillance. Open Access J Urol 2011; 4: 1-8.

3. Batool A, Karimi N, Wu XN, Chen SR, Liu YX. Testicular germ cell tumor: a comprehensive review. Cellular and Molecular Life Sciences. 2019 May 1;76(9):1713-27. https://doi.org/10.1007/s00018-019-03022-7.

4. Rajpert-De Meyts E, McGlynn KA, Okamoto $\mathrm{K}$, Jewett MA, Bokemeyer C. Testicular germ cell tumours. The Lancet. 2016 Apr 23;387(10029):1762-74. https://doi.org/10.1016/S0140-6736(15)009915 . 
5. Casey RG, Grainger R, Butler MR, McDermott TE, Thornhill JA. Public awareness of testis cancer and the prevalence of testicular selfexamination - changing patterns over 20 years. Urology. 2010 Oct 1;76(4):915-8. https://doi.org/10.1016/j.urology.2010.03.022.

6. Ondrusova M, Ondrus D. Epidemiology and treatment delay in testicular cancer patients: a retrospective study. Int Urol Nephrol. 2008 Mar;40(1):143-8. https://doi.org/10.1007/s11255-007-9245-3].

7. Williamson SR, Delahunt B, Magi-Galluzzi C, Algaba F, Egevad L, Ulbright TM, Tickoo SK, Srigley JR, Epstein JI, Berney DM, Members of the ISUP Testicular Tumour Panel. The World Health Organization 2016 classification of testicular germ cell tumours: a review and update from the International Society of Urological Pathology Testis Consultation Panel. Histopathology. 2017 Feb;70(3):335-46.

8. Milose JC, Filson CP, Weizer AZ, Hafez KS, Montgomery JS. Role of biochemical markers in testicular cancer: diagnosis, staging, and surveillance. Open Access J Urol 2011; 4: 1-8. https://doi.org/10.2147/OAJU.S15063.

9. Carrière P, Baade P, Fritschi L. Population based incidence and age distribution of spermatocytic seminoma. The Journal of urology. 2007 Jul;178(1):125-8.

10. Bernard B, Sweeney CJ. Diagnosis and treatment of testicular cancer: a clinician's perspective. Surgical pathology clinics. 2015 Dec 1;8(4):717-23, ISSN 1875-9181, ISBN 9780323395878, https://doi.org/10.1016/j.path.2015.07.006.

11. Vasdev N, Moon A, Thorpe AC. Classification, epidemiology and therapies for testicular germ cell tumours. International Journal of Developmental Biology. 2013 May 30;57(2-34):133-9.

12. Ahmed T, Bosl GJ, Hajdu SI. Teratoma with malignant transformation in germ cell tumors in men. Cancer. 1985 Aug 15;56(4):860-3.

13. Al-Hooti QM, Al-Mamari SA. Histological Classification of Testicular Cancer. InUrological Cancer Management 2015 (pp. 191-200). Springer, Cham. https://doi.org/10.1007/978-3-319-16301-7_18.

14. Kreydin EI, Barrisford GW, Feldman AS, Preston MA. Testicular cancer: what the radiologist needs to know. American Journal of Roentgenology. 2013 Jun;200(6):1215-25.

15. Sohaib SA, Koh DM, Husband JE. The role of imaging in the diagnosis, staging, and management of testicular cancer. American Journal of Roentgenology. 2008 Aug;191(2):387-95.

16. Corp IB. IBM SPSS statistics for windows, version 22.0. Armonk, NY: IBM Corp. 2013.

17. General Assembly of the World Medical Association. World Medical Association Declaration of Helsinki: ethical principles for medical research involving human subjects. The Journal of the American College of Dentists, 2014: 81(3), pp.14-18.

18. Leão R, Ahmad AE, Hamilton RJ. Testicular cancer biomarkers: a role for precision medicine in testicular cancer. Clinical genitourinary cancer. 2019 Feb 1;17(1):e176-83, ISSN 15587673 , https://doi.org/10.1016/j.clgc.2018.10.007.

19. Chovanec M, Albany C, Mego M, Montironi R, Cimadamore A, Cheng L. Emerging prognostic biomarkers in testicular germ cell tumors: looking beyond established practice. Front. Oncol. 2018 Nov 28;8:571. doi: 10.3389/fonc.2018.00571.

20. Rapley EA, Crockford GP, Teare D, Biggs P, Seal S, Barfoot R, Edwards S, Hamoudi R, Heimdal K, Fosså SD, Tucker K. Localization to Xq27 of a susceptibility gene for testicular germ-cell tumours. Nature genetics. 2000 Feb;24(2):197-200. 